\title{
The International Scientific Association for Probiotics and Prebiotics consensus statement on the scope and appropriate use of the term probiotic
}

\author{
Colin Hill, Francisco Guarner, Gregor Reid, Glenn R. Gibson, Daniel J. Merenstein, Bruno Pot, \\ Lorenzo Morelli, Roberto Berni Canani, Harry J. Flint, Seppo Salminen, Philip C. Calder \\ and Mary Ellen Sanders
}

\begin{abstract}
An expert panel was convened in October 2013 by the International Scientific Association for Probiotics and Prebiotics (ISAPP) to discuss the field of probiotics. It is now 13 years since the definition of probiotics and 12 years after guidelines were published for regulators, scientists and industry by the Food and Agriculture Organization of the United Nations and the WHO (FAO/WHO). The FAO/WHO definition of a probiotic- “live microorganisms which when administered in adequate amounts confer a health benefit on the host" - was reinforced as relevant and sufficiently accommodating for current and anticipated applications. However, inconsistencies between the FAO/WHO Expert Consultation Report and the FAO/WHO Guidelines were clarified to take into account advances in science and applications. A more precise use of the term 'probiotic' will be useful to guide clinicians and consumers in differentiating the diverse products on the market. This document represents the conclusions of the ISAPP consensus meeting on the appropriate use and scope of the term probiotic.
\end{abstract}

Hill, C. et al. Nat. Rev. Gastroenterol. Hepatol. 11, 506-514 (2014); published online 10 June 2014; doi:10.1038/nrgastro.2014.66

\section{Introduction}

In 2001, an Expert Consultation of international scientists working on behalf of the Food and Agriculture Organization of the United Nations (FAO) and the WHO debated the emerging field of probiotics. One output was a reworking of the definition of probiotics to the following: "live microorganisms which when administered in adequate amounts confer a health benefit on the host". Since then, this definition has become the most widely adopted and accepted version worldwide. In 2002, an FAO/WHO Working Group produced guidelines to assist with interpretation of the original document. ${ }^{2}$ Since the Expert Consultation report, PubMed has indexed $>8,000$ additional research articles that use the term probiotic. The scientific and clinical evidence have progressed rapidly, as has the development of a number of robust probiotic products. Unfortunately, misuse of the term probiotic has also become a major issue, with many products exploiting the term without meeting the requisite criteria. At the same time, probiotic products have received the legitimate attention of regulatory authorities with an interest in protecting consumers from misleading

Competing interests

C.H., G.R., G.R.G., S.S. and M.E.S. declare associations with the ISAPP. F.G., D.J.M., B.P., S.S., P.C.C. and M.E.S. declare associations with other companies and/or organizations. See the article online for full details of the relationships. R.B.C. and H.J.F. declare no competing interests. claims. Now, it is timely to revisit the concept of what can be defined as a probiotic, consider appropriate definitions and provide useful guidance for stakeholders.

The International Scientific Association for Probiotics and Prebiotics (ISAPP) organized a meeting of clinical and scientific experts on probiotics (with specialties in gastroenterology, paediatrics, family medicine, gut microbiota, microbiology of probiotic bacteria, microbial genetics, immunology and food science) held on 23 October 2013 to re-examine the concept of probiotics. Participants included members of the original FAO/WHO Expert Panel, members of the FAO/WHO Working Group and other internationally recognized experts. Participants in the meeting jointly considered key questions and generated and approved the outcomes hereby summarized.

We hope that this Consensus Statement will provide all probiotic stakeholders, including consumers, researchers, health-care professionals, industry and legislators, with clearer guidelines for defining and using probiotics, which we believe to be potentially important interventions for improved health and wellbeing. Throughout this paper, we use the term 'probiotic framework' to refer to all aspects of the probiotic field, including: scientific investigation and clinical research; regulatory involvement in safety, health benefit claims and research; industry activities including production, marketing, product claims and sales; and communication with the consumer. 
Box 1 | Consensus panel recommendations for the scope of probiotics

- Retain the FAO/WHO definition ${ }^{1}$ for probiotics, with a minor grammatical correction as "live microorganisms that, when administered in adequate amounts, confer a health benefit on the host"; inconsistences between the Expert Consultation ${ }^{1}$ and the FAO/WHO Guidelines ${ }^{2}$ were clarified

- Include in the framework for definition of probiotics microbial species that have been shown in properly controlled studies to confer benefits to health

- Any specific claim beyond "contains probiotics" must be further substantiated

- Keep live cultures, traditionally associated with fermented foods and for which there is no evidence of a health benefit, outside the probiotic framework

- Keep undefined, faecal microbiota transplants outside the probiotic framework

- New commensals and consortia comprising defined strains from human samples, with adequate evidence of safety and efficacy, are 'probiotics' Abbreviation: FAO, Food and Agriculture Organization of the United Nations.

Moreover, we propose a set of benchmark standards for the differentiation of probiotic products based upon levels of scientific evidence. Panel recommendations are listed in Box 1.

\section{Methods}

This panel was conceived by the board of directors of ISAPP. ISAPP is an international nonprofit collaboration of scientists dedicated to advancing scientific excellence in probiotics and prebiotics. ISAPP's activities are determined by the board of directors, comprising global academic scientists. Through its Industry Advisory Committee, ISAPP incorporates industry scientists in its activities and raises funds. ISAPP strives to be an objective, science-based voice for the probiotic and prebiotic fields.

To prepare for the panel, experts were selected from across a range of relevant disciplines, including current board members as well as external experts. Panellists developed a discussion outline and target questions. Several panellists (C.H., B.P., G.R., F.G., M.E.S. and L.M.) delivered brief presentations that addressed the background and core issues involved for each question. Discussion ensued for each issue until a unanimous consensus was achieved. After the meeting, individual panellists wrote sections of the summary, which were compiled into a draft report. This document was circulated and agreed before submission.

\section{Revisiting the term 'probiotic'}

'Probiotic' is a useful and accepted term. The FAO/ WHO definition has been widely adopted and has proven valuable to researchers, regulators and consumers. Organizations and agencies such as Codex (which comes under the FAO/WHO umbrella), Health Canada, ${ }^{3}$ the World Gastroenterology Organisation, ${ }^{4}$ the European Food Safety Authority (EFSA) and the Institute of Food Technologists ${ }^{5}$ use the FAO/WHO definition when referring to probiotics. The panel noted, however, that a more grammatically correct definition would be worded as, "live microorganisms that, when administered in adequate amounts, confer a health benefit on the host" and supports use of this wording going forward. This definition is inclusive of a broad range of microbes and applications, whilst capturing the essence of probiotics (microbial, viable and beneficial to health). The definition differentiates live microbes used as processing aids or sources of useful compounds from those that are administered primarily for their health benefits. ${ }^{6}$ The distinction between commensal microorganisms and probiotics is also inferred from this definition. Although commensals in the gut are often the source of probiotic strains, until these strains are isolated, characterized and a credible case presented for their health effects, they cannot be called 'probiotics'. In the 13 years that have passed since the definition was proposed, numerous lines of research have challenged the limits of the probiotic concept, from live cultures present in fermented foods to faecal microbiota transplants (FMT). The term has also been clearly misused, for example, on products such as mattresses, shampoos, disinfectants and aftershave, for which maintenance of viability and efficacy of the microbes used are not established. Use of the term probiotic has been restricted in some countries of the European Union because it is deemed misleading to consumers in the absence of approved health claims. ${ }^{7}$ It is now evident that different interpretations of the term probiotic are creating notable concerns for major stakeholders with respect to the translation of a large body of research on probiotics to probiotic-containing foods that can benefit consumers. The objectives of the different stakeholders in the probiotic field are described in Figure 1; notably, all the stakeholders' objectives are compatible. Importantly, all parties involved in the probiotic field must work toward a common goal so that society benefits from the scientific advances in the field of probiotic research. These concerns motivated the ISAPP to convene a panel of experts to address the following relevant questions with respect to defining the term probiotic.

\section{(1) Are there core benefits that can be ascribed to the general category of probiotics, and, if so, should the probiotic classification include live microbes identified to the species level, which can be reasonably expected to impart general benefits?}

\section{Probiotic benefits}

On the basis of the currently available literature, which includes well-designed clinical trials, systematic reviews and meta-analyses, the consensus panel concurred that certain effects can be ascribed to probiotics as a general class. In this context, we refer to strains of a number of well-studied microbial species delivered at a functional dose for use as foods or supplements in the general population-not strains used as drugs. This opinion aligns with regulatory approaches in Canada and Italy. Health Canada has accepted the following bacterial species, when delivered in food at a level of $1 \times 10^{9}$ colony forming units (CFU) per serving, as probiotics for which nonstrain-specific claims might be made: Bifidobacterium (adolescentis, animalis, bifidum, breve and longum) and Lactobacillus (acidophilus, casei, fermentum, gasseri, johnsonii, paracasei, plantarum, rhamnosus and salivarius). ${ }^{3}$ This list represents a core group of well-studied species likely to impart some general benefits. Acceptable claims on Canadian foods 


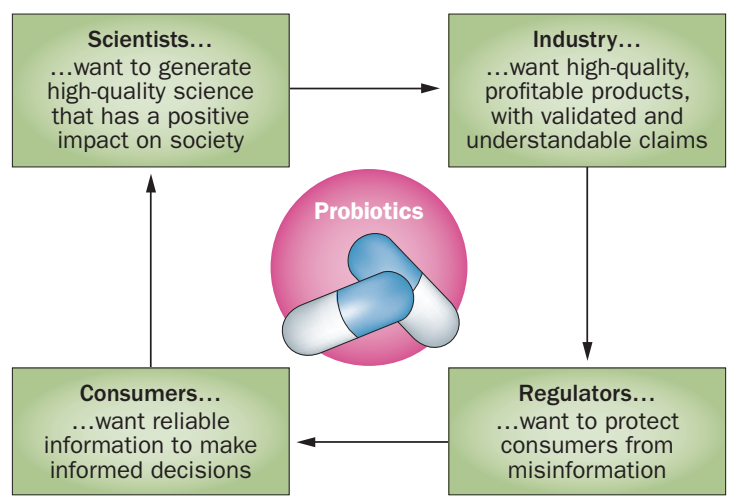

Figure 1 | Objectives of stakeholders in the probiotic field. These objectives are compatible, so there is no apparent reason for obstruction of probiotic product development and marketing. Importantly, all stakeholders must work together performing their respective duties so that society benefits from the scientific advances in the field of probiotic research.

for these probiotics are based on their contribution to a healthy gut microbiota. Similarly, nutrition recommendations in some European Union countries suggest the consumption of specific species for nutrition and health benefits. ${ }^{8}$ For more than 30 years, Italy has had a tradition of using beneficial bacteria, administered as food supplements or food ingredients, to help manage the intestinal microbiota. The Italian Ministry of Health has regulated the use of probiotic bacteria in the food sector over the past 12 years and, in 2013, confirmed the use of the word probiotic for food and food supplements under certain conditions, including a minimum number of viable cells $\left(1 \times 10^{9} \mathrm{CFU}\right)$ administered per day, a full genetic characterization of the probiotic strain and a demonstratable history of safe use in the Italian market. ${ }^{9}$

Thus, Canada and Italy consider the general benefit of supporting a healthy gut microbiota to be a core benefit of probiotics. The consensus panel agrees with this approach, while acknowledging that the current state of science does not allow the clear definition of a healthy gut microbiota based on microbial composition. ${ }^{10}$ Nevertheless, the general benefit of probiotics on gut microbiota derives from creating a more favourable gut environment, through mechanisms shared by most probiotics. The panel further considered two common general benefits often associated with probiotics: supporting a healthy digestive tract and a healthy immune system. The panel concluded that the general benefit of supporting a healthy digestive tract was reinforced by evidence gathered on a large number of different probiotic strains representing commonly studied species. This conclusion was based on a body of available research, including high-quality meta-analyses, on a diversity of clinical end points (such as infectious diarrhoea, antibiotic-associated diarrhoea, gut transit, IBS, abdominal pain and bloating, ulcerative colitis and necrotizing enterocolitis ${ }^{11-15}$ ), as well as potential mechanistic actions suggesting that most strains of these species can be expected to have such 'generic' or 'core' effects on gut physiology and health. The core benefit of supporting a healthy immune system was considered by the panel to be widely acknowledged, but probably more strain-specific. ${ }^{16,17}$ The diverse meaning of 'supporting a healthy immune system', which ranges from preventing allergic disease to downregulation of inflammation to the enhancement of anti-infection activities was considered to be too broad to be considered a core benefit. Other benefits such as supporting the health of the reproductive tract, oral cavity, lungs, skin and gut-brain axis are promising, but evidence has not yet been linked to a broad enough cross-section of probiotics to consider these effects to be probably shared across the whole class of probiotics.

\section{Underlying mechanisms}

The panel considered the current understanding of the mechanisms underlying probiotic effects. Figure 2 considers, in a general sense, the distribution of mechanisms among probiotic strains. Although specific attributions can be debated, the key point is that some mechanisms are widespread among a diversity of strains whereas others are less so. Widespread mechanisms can be associated with effects that are observed across taxonomic groups, such as inhibition of potential pathogens or the production of useful metabolites or enzymes. ${ }^{18,19}$ Other effects at the intestinal or extraintestinal level, including immune effects, are more likely to be strain-specific ${ }^{20}$ and claims of such benefit can only be made for strains or species in which the mechanistic basis has been demonstrated. ${ }^{21,22}$ In many cases, a given probiotic might exert several healthpromoting effects. Although multiple mechanisms are often represented in a single strain, no individual probiotic would be expected to have all the effects listed in Figure 2.

\section{Core benefits}

The panel is convinced that sufficient evidence has accumulated to support the concept of 'core' benefits of certain probiotics; it is reasonable to expect that evidence gained from a defined class of live microbes might be appropriate for certain, but not all, health outcomes. This stance is in contrast to a prevailing perspective that every probiotic strain is different and probably elicits a different outcome in the host. We conclude that this morenuanced understanding of probiotics is justified based on accumulated evidence from the hundreds of human studies and dozens of positive meta-analyses available today. It seems remarkable that probiotic strains selected decades ago for use in commercial probiotic products (in which strain robustness, growth and stability would have been the central criteria) have proven to be effective in conferring a health benefit in multiple trials with various end points. This finding supports the concept that there are common health benefits to be derived from consuming (or delivering) at an adequate dose any safe strain of a species that is already known to include an effective probiotic. For example, if the hypothesis under investigation was "safe bacterial cultures administered in high doses will have a beneficial outcome in gastrointestinal diseases", then the appropriate methodology would be to select a wide range of individual bacterial 


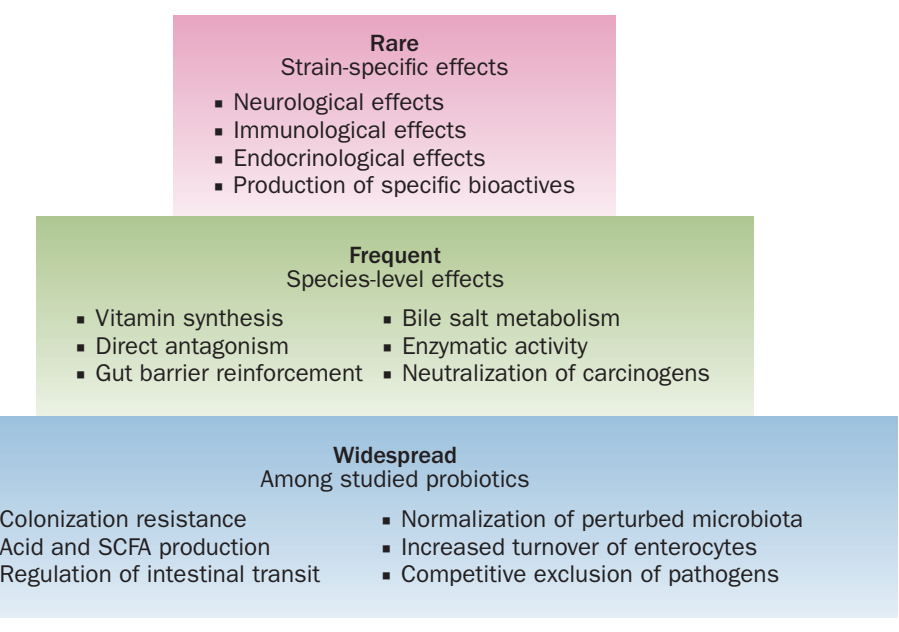

Figure 2 | Possible distribution of mechanisms among probiotics. Some mechanisms might be widespread among commonly studied probiotic genera; others might be frequently observed among most strains of a probiotic species; others may be rare and present in only a few strains of a given species. Evidence is accumulating on a cross-section of probiotic strains that suggest some generalizations can be made beyond strain-specific effects. Abbreviation: SCFA, short-chain fatty acid.

strains or strain mixes, and test them in multiple settings in diverse patient groups. The outcome of all of the individual trials would then be analysed collectively. This approach is essentially what has been done in numerous meta-analyses conducted on multiple probiotic strains that have been used in human trials.

One such meta-analysis was performed by Ritchie and Romanuk, ${ }^{11}$ which included a broad range of probiotic strains; null studies were also part of the analysis. In their meta-analysis containing 74 studies, 84 trials and 10,351 patients, the authors concluded that, in general, probiotics are beneficial in the treatment and prevention of gastrointestinal diseases. This approach of combining probiotic strains of various genera and species into one functional 'class' has already been accepted by EFSA for yogurt cultures, as evidenced by an approved claim for Lactobacillus delbrueckii subsp. bulgaricus and Streptococcus salivarius subsp. thermophilus in aiding lactose digestion. ${ }^{23}$ The EFSA, in this instance, accepted characterization at the species-not strain-level because the mechanism of action is clearly understood as being microbial production of $\beta$-galactosidase, which aids lactose digestion in the gut. However, the claimant does not have to demonstrate that every strain of L. delbruueckii subsp. bulgaricus or S. salivarius subsp. thermophilus produces sufficient lactase to support the claim, and so the benefit is ascribed to this class of microbe. Similarly, whereby a core benefit could be associated with a particular structure or activity, it would be reasonable to use data accumulated for any strain exhibiting that property as support for a health claim. It would also seem equitable to allow the use of the term 'probiotic' for a member of a species for which systematic reviews or meta-analyses indicate a general health benefit, particularly (or perhaps only) if a specific health claim was not being communicated on the product.
Should the probiotic framework include traditional fermented foods containing live microbes?

The panel acknowledged that evidence supports the beneficial relationship between some foods containing live microbes, especially fermented dairy products, and reduced risk of certain diseases. For example, fermented dairy products have been associated with reduced risk of type 2 diabetes or improved markers of glucose homeostasis, ${ }^{24-26}$ less weight gain over time in a prospective study of $>120,000$ adults $^{27}$ and reduced risk of overall mortality. ${ }^{28}$ An observational study of $>6,500$ individuals found that yogurt consumers had reduced levels of circulating triglycerides and glucose, as well as reduced systolic blood pressure and insulin resistance, compared with nonconsumers. ${ }^{29}$ Consumption of foods containing live microbes might therefore be a beneficial dietary recommendation. However, in the panel's judgement, it is not always possible to clearly distinguish the contribution of the live microbes from that of the food matrix in such studies. Furthermore, potentially beneficial microbes might often represent a diverse community that is not well-defined in terms of strain composition and stability. As a result, the live microbes in such foods fall short of the criteria needed to be considered 'probiotics'. It was recommended that such foods are best described as 'containing live and active cultures', but should not be called probiotic.

\section{Q Should the probiotic framework include well-defined beneficial commensal microbes?}

Research has suggested that certain individual constituents of the human microbiota might represent novel candidates for new probiotics. Metagenomic studies provide a profile of the microbial communities in the human gut and reveal an association between low microbial diversity and several disease states, including IBD, metabolic disorders and IBS. ${ }^{30-36}$ Even if a cause-effect relationship between low microbial richness and disease has not been established, it is known that exposure to antibiotics early in life is a risk factor for the development of $\mathrm{IBD}^{37}$ or obesity, ${ }^{38}$ suggesting that depletion of some critical commensals might have health consequences later in life. These observations, among others, support the hypothesis that the increasingly vulnerable human microbiota and specifically defective colonization by 'old friends' play a part in the aetiology of a number of disorders of modern society. ${ }^{39,40}$ Hypothetically, replacement of the missing commensal microbes with individual strains or defined strain mixes could help in the prevention and treatment of such disorders.

Metagenomic studies of the human microbiome have identified a number of genera and species that are associated with a robust gut microbiota composition, and experimental work in animal models of disease has provided evidence that some of them might be useful tools to mitigate intestinal inflammation, ${ }^{41}$ induce immune regulation, ${ }^{42}$ or enhance intestinal barrier function. ${ }^{43}$ These microbes include Akkermansia muciniphila ${ }^{44}$ and Faecalibacterium prausnitzii ${ }^{45}$ together with other butyrate-producing bacteria such as Roseburia spp. ${ }^{46}$ and 
Eubacterium hallii. ${ }^{47}$ Whether these microbes can be used in foods, dietary supplements, medical foods, medical devices or drugs will depend on demonstration of safety and efficacy for these uses and within regulatory frameworks. The panel considered that such microbes clearly fall under the term probiotic, as long as suitable safety assessments are conducted. ${ }^{6}$ For these new probiotics, which might comprise little-studied species, it would seem advisable to proceed on a strain-by-strain basis until such time that there is sufficient information and mechanistic understanding for extrapolation to the species level.

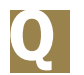

Should the probiotic framework include undefined consortia of commensals derived from sampling human body sites?

The panel acknowledged the increased use of undefined mixtures of microbes derived from human samples to treat gastrointestinal diseases through FMT. ${ }^{48}$ To date, most clinical experience has focused on the use of FMT in patients with relapsing diarrhoea due to Clostridium difficile infection, in which the procedure resolves recurrence in $~ 90 \%$ of cases. ${ }^{49}$ Interest also exists in the therapeutic potential of FMT for IBD, IBS and some extraintestinal conditions such as metabolic and autoimmune diseases. ${ }^{48}$ The safety (especially in the long term) of such procedures remains to be established. ${ }^{50}$ Donor FMT mixtures can comprise a number of unknown taxa, including bacteria, yeasts, parasites and viruses, and it is not known which microbes are responsible for the beneficial effect, and which might pose a risk through transfer of antibiotic resistance, production of genotoxic metabolites or intestinal translocation. In addition, FMT cannot be standardized, even with the same donor, due to inter-individual and intra-individual variability of the gut microbiota. ${ }^{51}$ Nevertheless, regulatory authorities in the USA, which initially banned all clinical use of FMT except under an investigational new drug application, reversed course and now allow clinical use for treatment of $C$. difficile disease. ${ }^{52}$

The lack of identification of the microbes being used from a wide range of donors motivated the panel to exclude FMT from the probiotic framework. In a similar manner, undefined collections of microbes from other human body sites are not considered probiotics. However, the panel determined that preparations of defined mixes of microbes such as 'RePoop, ${ }^{53}$ in an approach coined 'microbial ecosystems therapeutics' by Petrof and colleagues, ${ }^{54} \mathrm{did}$ meet the criteria of a probiotic, as long as the microbial preparations comprise well-defined microbial components.

[Q]

Should the FAO/WHO definition and guidelines continue to be endorsed?

Thus far we have described examples of how different microbes meet the FAO/WHO definition of a probiotic. The panel determined that this definition remains relevant and useful for scientists and regulators to define the broad category of probiotics. Furthermore, we considered that the FAO/WHO Guidelines were also relevant, with one modification. These Guidelines state:
"Use and adoption of the guidelines in this report should be a prerequisite for calling a bacterial strain probiotic". The panel considered that due to the accumulated evidence, general benefits can be reasonably associated with well-researched probiotic species, to the extent that even if randomized controlled trials (RCTs) are not conducted on specific strains, they might still be considered as 'probiotics'. In addition, the section of the Guidelines addressing genetic characterization of probiotics should be updated to include the use of genomic sequencing as part of a thorough safety assessment.

Other inconsistencies between documents were noted. The purpose of the Expert Consultation and Working Group, convened by the FAO/WHO in $2001^{1}$ and 2002, ${ }^{2}$ respectively, was to establish guidelines for assessing the efficacy of probiotics delivered as food. However, numerous examples of probiotic delivery that extend beyond food applications appear in these documents. For example, the probiotic definition adopted by the Expert Consultation clearly was not limited to probiotic foods, and indeed included non-oral applications. The word 'administered' was deliberately chosen rather than 'consumed' and no limits were placed on the type of health effects or target populations in the definition, thereby allowing many applications to be feasible. Most of the evidence listed for probiotic beneficial effects dealt with pathological conditions, which correctly belong in the realm of drug applications as currently differentiated by most regulatory agencies. Another inconsistency between the two documents is that the Expert Consultation ${ }^{1}$ stated that the ability to remain viable at the target site should be verified for each potential strain. This point was not included in the Guidelines. ${ }^{2}$ The Expert Consultation further states that "...traditional starters are not to be considered probiotics since they lack the ability to proliferate in the intestine". However, the 2002 Guidelines recognized probiotic status for traditional yogurt cultures (S. salivarius subsp. thermophilus and L. delbrueckii subsp. bulgaricus) owing to their ability to improve tolerance to lactose in maldigesters. The beneficial effects of these cultures have since been endorsed by the EFSA. ${ }^{23}$ Certainly, other health benefits that do not require a strain to proliferate in the intestine are conceivable and are within the scope of probiotics.

As would be expected given the rapidity of scientific breakthroughs, the research regarding the mechanisms and health effects of probiotics extend much beyond what was included in the 2001 Expert Consultation. ${ }^{1}$ Nevertheless, the definition remains relevant, and the Guidelines document still provides a useful approach to validating a probiotic strain.

What is an appropriate level of evidence for determining a health benefit for probiotics?

To determine whether an association exists between a substance (such as a probiotic) and a desired outcome (such as maintaining a healthy digestive system), it is important to examine the following criteria: temporal relationship; strength of relationship; dose response; replication of findings; biological plausibility; consideration 
Table 1 | Categories of live microorganisms for human use as defined by the expert panel

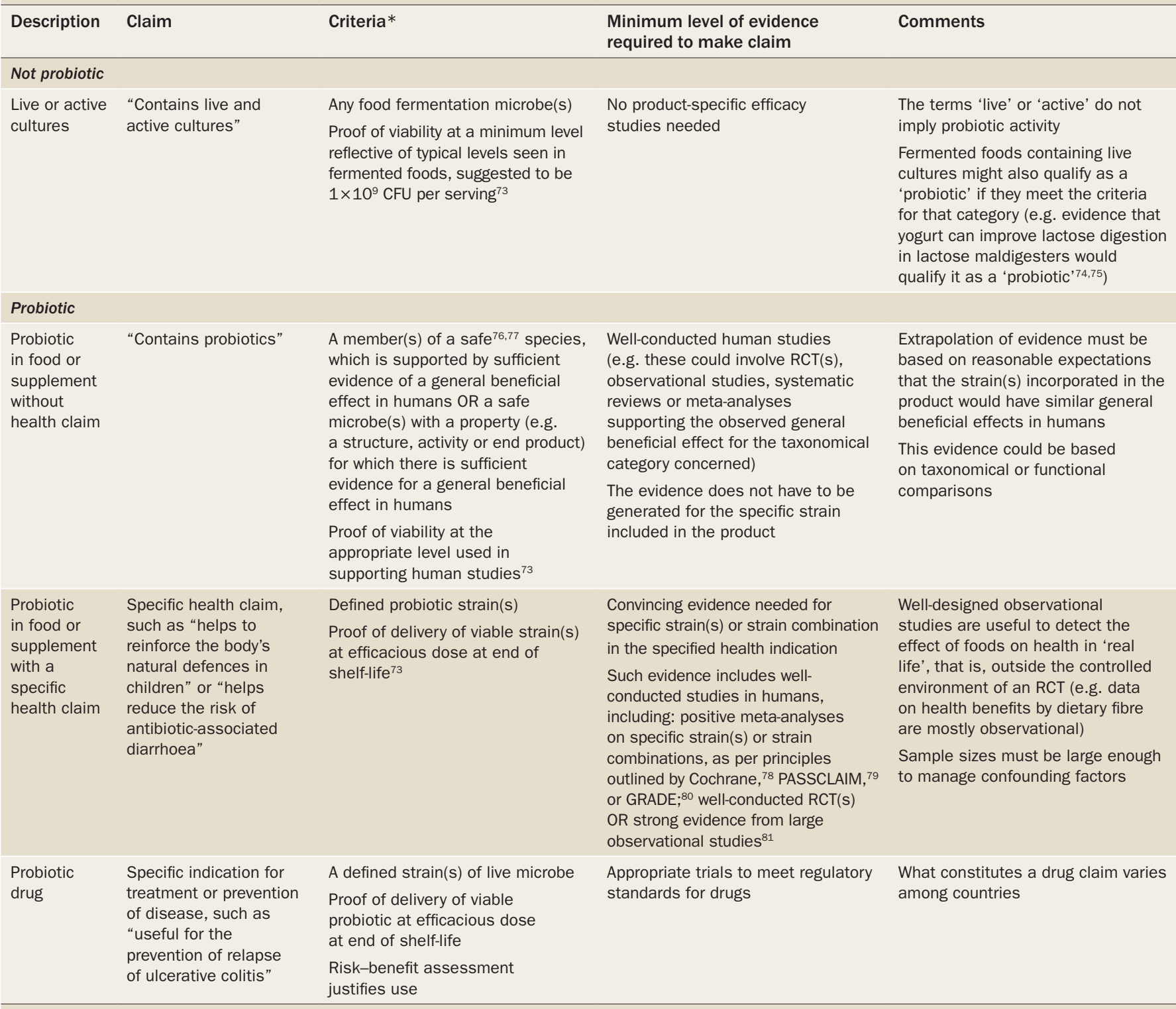

*Unless otherwise indicated, all criteria indicated must be met. Abbreviations: CFU, colony forming unit; GRADE, Grades of Recommendation Assessment, Development and Evaluation; PASSCLAIM, Process for the Assessment of Scientific Support for Claims on Food; RCT, randomized controlled trial.

of alternative explanations; cessation of exposure; specificity of the association; and consistency with other knowledge. ${ }^{55,56}$ Subtle discrepancies exist among different epidemiological authorities but these criteria (based on the Bradford Hill Criteria) are important factors for determining a causal relationship. Appropriately powered, well-designed RCTs, preferably assessed through the systematic review and meta-analysis process, provide the evidence to draw conclusions on causality. However, such studies might not be available for determining strength of evidence to substantiate health-benefit claims for dietary (nutritional) supplements or foods.

The panel also believes that probiotic foods or supplements should not be held to a higher standard of evidence than other foods or supplements. No probiotic claims have been judged sufficiently substantiated for foods in the European Union. Evidence that vitamin C reduced tiredness and fatigue, contributed to normal energyyielding metabolism and maintained the normal function of the immune system was deemed sufficient, ${ }^{57}$ yet there are no robust RCTs in healthy individuals supporting these benefits-the importance of dietary vitamin $\mathrm{C}$ is based on malnourished patients who have developed scurvy. ${ }^{58-61}$ Many other supplements are also recommended by doctors for uses not supported by RCTs. For example, for bone health, many primary-care physicians recommend supplementation with calcium and vitamin D, yet limited RCT evidence exists to support this recommendation. Indeed, a Cochrane review found that evidence does not support vitamin D supplementation to improve bone density in healthy children. ${ }^{62}$

Health effects of probiotics are supported by many wellconducted RCTs and high-quality systematic reviews and meta-analyses. ${ }^{14,63-66}$ That the totality of evidence must be 


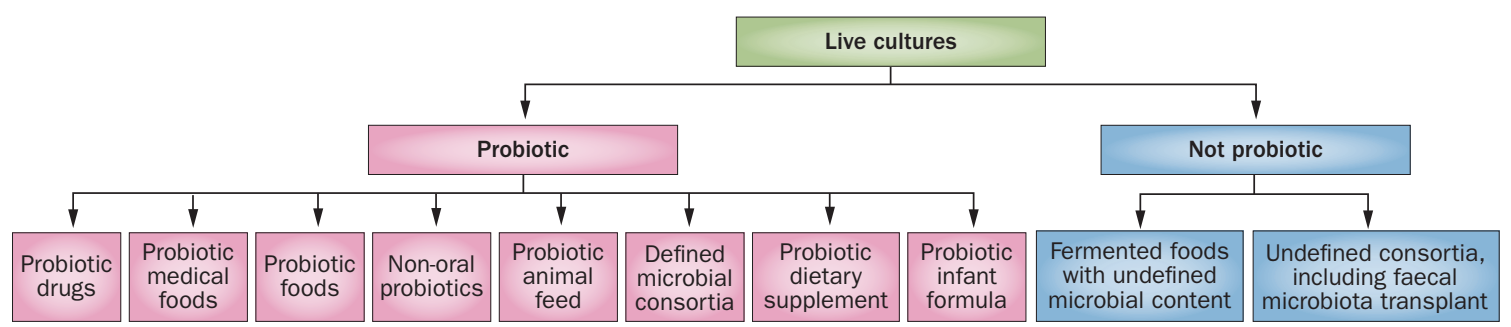

Figure 3 | Overall framework for probiotic products. Evidence of a health benefit is required for a probiotic, at either a strain-specific or group level, depending on the nature of the benefit. Probiotics can have different means of administration, target host species (humans and animals), target populations, target sites (gut and beyond), efficacy end points and regulatory categories. All probiotics must be safe for their intended use. Dead microbes, microbial products, microbial components do not come under the probiotic classification.

considered when evaluating evidence for health benefits is widely accepted. The panel urges a consistent approach be taken to clinical, food and dietary recommendations by health-care professionals and regulators.

\section{Implications of the recommendations}

The marketplace has many products carrying the label 'probiotic', but too often they do not meet minimum criteria, such as defined contents, appropriate viable count at end of shelf-life and suitable evidence for health benefit. The panel recommends that the term probiotic be used only on products that deliver live microorganisms with a suitable viable count of well-defined strains with a reasonable expectation of delivering benefits for the wellbeing of the host (Table 1).

In addition, there is a need for clear communication to consumers and health-care providers on how to differentiate probiotic products. Unfortunately, this goal is difficult to achieve because of regulatory agency restrictions on what can be communicated on product labels. Restricting use of the term probiotic on foods, as has occurred in some countries in the European Union, is unwarranted when minimum criteria are met. A product-labelling approach that communicates the information known about the probiotic being sold would be more useful for consumers and health-care providers.

This panel, or any other panel of experts, cannot force change and, ultimately, the marketplace and regulators will decide which probiotics are made available for consumers. However, as supporters of the importance of translating the benefits of rigorous scientific and medical research, we reiterate that studies have shown, and continue to show, that certain types of microorganisms are beneficial to human health, and we suggest that limiting consumer access to this information has negative implications for the health of adults and children around the world.

\section{Conclusions}

The panel believes its recommendations are important for several key reasons. Research advances over the past 12 years warrant discussion about the relevance of the probiotic concept. ${ }^{67-69}$ The panel found that the definition of a probiotic advanced by the FAO/WHO in 2001 is sufficiently broad to enable a wide range of products to be developed, and sufficiently narrow to impose some core requirements (Table 1). The development of metabolic by-products, dead microorganisms, or other microbialbased, nonviable products has potential; however, these do not fall under the probiotic construct. Figure 3 represents an overall framework for use of the term 'probiotic', encompassing diverse end uses. Substantial confusion exists in the regulation of probiotics, in many cases because regulators are attempting to apply schemes to probiotic foods and supplements that were initially designed to facilitate pharmaceutical development. We concur with a report published in $2013^{70}$ that changes are needed to how probiotics are handled by regulators in jurisdictions such as the USA and Europe. However, we disagree strongly with the regulatory action by the FDA in the USA to require investigational new drug applications for all functional end points for probiotic foods and for most dietary supplements. ${ }^{71}$ This action will severely hinder the conduct and increase the cost of safe, much-needed research on probiotics, through unnecessary bureaucratic requirements that are difficult, if not impossible, to meet for products not manufactured using pharmaceutical standards. The drug approach to research can add to the cost and delay needed efficacy studies by requiring safety studies not considered necessary by Institutional Review Boards overseeing the research. The panel identifies a need to improve communication to the public and health-care professionals on the benefits of probiotics, which can be expected for the general population. The panel also acknowledges that robust evidence must be provided for benefits tied to specific strains. This stance is similar to a category system originally proposed in $2012,{ }^{67}$ but laid out clearly for all stakeholders.

The field of probiotics has advanced considerably in recent years, spurred by global progress in understanding the role of the human microbiota in health and disease and the need to define effective strategies to shape a healthier microbiota. Well-controlled intervention trials, systematic reviews and meta-analyses provide convincing evidence of the benefits of probiotics, including ones with valuable public health implications ${ }^{68,72}$ Clarifying the proper scope and appropriate use of the term probiotic is important so that all stakeholders in the probiotic field, as depicted in Figure 1, share an understanding of probiotics that is consistent with current research. This clarification will facilitate continued advances in probiotic research and will ensure probiotic benefits are properly communicated to consumers and patients. 
1. Food and Agricultural Organization of the United Nations and World Health Organization. Health and nutritional properties of probiotics in food including powder milk with live lactic acid bacteria. World Health Organization [online], http://www.who.int/foodsafety/publications/ fs_management/en/probiotics.pdf (2001).

2. Food and Agricultural Organization of the United Nations and World Health Organization. Joint $\mathrm{FAO} / \mathrm{WHO}$ working group report on drafting guidelines for the evaluation of probiotics in food. Food and Agricultural Organization of the United Nations [online], ftp://ftp.fao.org/es/ esn/food/wgreport2.pdf (2002).

3. Health Canada. Accepted Claims about the Nature of Probiotic Microorganisms in Food. Health Canada [online], http://www.hc-sc.gc.ca/ fn-an/label-etiquet/claims-reclam/probiotics claims-allegations probiotiques-eng.php\%20 (2009).

4. Guarner, F. et al. World Gastroenterology Organisation Global Guidelines: probiotics and prebiotics October 2011. J. Clin. Gastroenterol. 46, 468-481 (2012).

5. Ohr, L. M. Health benefits of probiotics and prebiotics. Institute of Food Technologists [online], http://www.ift.org/food-technology/ past-issues/2010/march/columns/ nutraceuticals.aspx?page=viewall (2010).

6. Sanders, M. E. How do we know when something called "probiotic" is really a probiotic? A guideline for consumers and health care professionals. Functional Food Rev. 1, 3-12 (2009).

7. Food Safety Authority of Ireland. Probiotic Health Claims. Food Safety Authority of Ireland [online], http://www.fsai.ie/fags/probiotic health claims.html (2013).

8. Smug, L. N., Salminen, S., Sanders, M. E. \& Ebner, S. Yoghurt and probiotic bacteria in dietary guidelines of the member states of the European Union. Benef. Microbes 5, 61-66 (2014).

9. Ministero della Salute, Commissione unica per la nutrizione e la dietetica. Guidelines on probiotics and prebiotics. Ministero della Salute [online], http://www. salute.gov.it/imgs/ C 17 pubblicazioni 1016 allegato.pdf (2013).

10. Backhed, F. et al. Defining a healthy human gut microbiome: current concepts, future directions, and clinical applications. Cell Host Microbe 12 , 611-622 (2012).

11. Ritchie, M. L. \& Romanuk, T. N. A meta-analysis of probiotic efficacy for gastrointestinal diseases. PLoS ONE 7, e34938 (2012).

12. Bernaola Aponte, G., Bada Mancilla, C. A. Carreazo, N. Y. \& Rojas Galarza, R. A. Probiotics for treating persistent diarrhoea in children. Cochrane Database of Systematic Reviews, Issue 8. Art. No.: CD007401 http://dx.doi.org/ 10.1002/14651858.CD007401.pub3.

13. Goldenberg, J. Z. et al. Probiotics for the prevention of Clostridium difficile-associated diarrhea in adults and children. Cochrane Database of Systematic Reviews, Issue 5. Art. No.: CD006095 http://dx.doi.org/ 10.1002/14651858.CD006095.pub3.

14. Alfaleh, K., Anabrees, J., Bassler, D. \& Al-Kharfi, T. Probiotics for prevention of necrotizing enterocolitis in preterm infants. Cochrane Database of Systematic Reviews, Issue 3. Art. No.: CD005496 http://dx.doi.org/ 10.1002/14651858.CD005496.pub3.

15. Allen, S. J., Martinez, E. G., Gregorio, G. V. \& Dans, L. F. Probiotics for treating acute infectious diarrhoea. Cochrane Database of Systematic Reviews Issue 11. Art. No. CD003048 http://dx.doi.org/10.1002/ 14651858.CD003048.pub3.
16. Lomax, A. R. \& Calder, P. C. Probiotics, immune function, infection and inflammation: a review of the evidence from studies conducted in humans. Curr. Pharm. Des. 15, 1428-1518 (2009).

17. Maidens, C., Childs, C., Przemska, A., Dayel, I. B. $\&$ Yaqoob, P. Modulation of vaccine response by concomitant probiotic administration. Br. J. Clin. Pharmacol. 75, 663-670 (2013).

18. Kumar, M. et al. Probiotic metabolites as epigenetic targets in the prevention of colon cancer. Nutr. Rev. 71, 23-34 (2013).

19. Reid, G. et al. Microbiota restoration: natural and supplemented recovery of human microbia communities. Nature Rev. Microbiol 9, 27-38 (2011).

20. van Baarlen, P. et al. Human mucosal in vivo transcriptome responses to three lactobacilli indicate how probiotics may modulate human cellular pathways. Proc. Natl Acad. Sci. USA 108 (Suppl. 1), 4562-4569 (2011).

21. Heuvelin, E. et al. Mechanisms involved in alleviation of intestinal inflammation by Bifidobacterium breve soluble factors. PLOS ONE 4, e5184 (2009)

22. Macho Fernandez, E. et al. Anti-inflammatory capacity of selected lactobacilli in experimental colitis is driven by NOD2-mediated recognition of a specific peptidoglycan-derived muropeptide. Gut 60, 1050-1059 (2011).

23. EFSA Panel on Dietetic Products, Nutrition and Allergies. Scientific Opinion on the substantiation of health claims related to live yoghurt cultures and improved lactose digestion (ID 1143, 2976) pursuant to Article 13(1) of Regulation (EC) No 1924/2006. EFSA Journal 8 , 1763 (2010).

24. Tong, X., Dong, J. Y., Wu, Z. W., Li, W. \& Qin, L. Q. Dairy consumption and risk of type 2 diabetes mellitus: a meta-analysis of cohort studies. Eur. J. Clin. Nutr. 65, 1027-1031 (2011).

25. Struijk, E. A. et al. Dairy product intake in relation to glucose regulation indices and risk of type 2 diabetes. Nutr. Metab. Cardiovasc. Dis. 23, 822-828 (2013).

26. Sluijs, I. et al. The amount and type of dairy product intake and incident type 2 diabetes: results from the EPIC-InterAct Study. Am. J. Clin. Nutr. 96, 382-390 (2012).

27. Mozaffarian, D., Hao, T., Rimm, E. B., Willett, W. C. \& Hu, F. B. Changes in diet and lifestyle and longterm weight gain in women and men. $N$. Engl. J. Med. 364, 2392-2404 (2011).

28. Soedamah-Muthu, S. S., Masset, G., Verberne, L., Geleijnse, J. M. \& Brunner, E. J. Consumption of dairy products and associations with incident diabetes, CHD and mortality in the Whitehall II study. Br. J. Nutr. 109, 718-726 (2013).

29. Wang, H., Livingston, K. A., Fox, C. S., Meigs, J. B. $\&$ Jacques, P. F. Yogurt consumption is associated with better diet quality and metabolic profile in American men and women. Nutr. Res. 33, 18-26 (2013).

30. Qin, J. et al. A human gut microbial gene catalogue established by metagenomic sequencing. Nature 464, 59-65 (2010).

31. Lepage, P. et al. Twin study indicates loss of interaction between microbiota and mucosa of patients with ulcerative colitis. Gastroenterology 141, 227-236 (2011).

32. Manichanh, C., Borruel, N., Casellas, F. \& Guarner, F. The gut microbiota in IBD. Nat. Rev. Gastroenterol. Hepatol. 9, 599-608 (2012).

33. Qin, J. et al. A metagenome-wide association study of gut microbiota in type 2 diabetes. Nature 490, 55-60 (2012).

34. Le Chatelier, E. et al. Richness of human gut microbiome correlates with metabolic markers. Nature 500, 541-546 (2013).
35. Jeffery, I. B. et al. An irritable bowel syndrome subtype defined by species-specific alterations in faecal microbiota. Gut 61, 997-1006 (2012).

36. Rajilic-Stojanovic, M. et al. Global and deep molecular analysis of microbiota signatures in fecal samples from patients with irritable bowe syndrome. Gastroenterology 141, 1792-1801 (2011).

37. Hviid, A., Svanstrom, H. \& Frisch, M. Antibiotic use and inflammatory bowel diseases in childhood. Gut 60, 49-54 (2011).

38. Trasande, L. et al. Infant antibiotic exposures and early-life body mass. Int. J. Obes. (Lond.) 37 , 16-23 (2013).

39. Guarner, F. et al. Mechanisms of disease: the hygiene hypothesis revisited. Nat. Clin. Pract. Gastroenterol. Hepatol. 3, 275-284 (2006).

40. Blaser, M. J. \& Falkow, S. What are the consequences of the disappearing human microbiota? Nat. Rev. Microbiol. 7, 887-894 (2009).

41. Sokol, H. et al. Faecalibacterium prausnitzii is an anti-inflammatory commensal bacterium identified by gut microbiota analysis of Crohn disease patients. Proc. Natl Acad. Sci. USA 105 16731-16736 (2008).

42. Atarashi, K. et al. Treg induction by a rationally selected mixture of clostridia strains from the human microbiota. Nature 500, 232-236 (2013).

43. Everard, A. et al. Cross-talk between Akkermansia muciniphila and intestinal epithelium controls diet-induced obesity. Proc. Natl Acad. Sci. USA 110, 9066-9071 (2013).

44. Joyce, S. A. \& Gahan, C. G. The gut microbiota and the metabolic health of the host. Curr. Opin. Gastroenterol. 30, 120-127 (2014).

45. Miquel, S. et al. Faecalibacterium prausnitzii and human intestinal health. Curr. Opin. Microbiol. 16 , 255-261 (2013).

46. Louis, P., Scott, K. P., Duncan, S. H. \& Flint, H. J. Understanding the effects of diet on bacterial metabolism in the large intestine. J. Appl. Microbiol. 102, 1197-1208 (2007).

47. Vrieze, A. et al. Transfer of intestinal microbiota from lean donors increases insulin sensitivity in individuals with metabolic syndrome. Gastroenterology 143, 913-916 (2012).

48. Smits, L. P., Bouter, K. E., de Vos, W. M., Borody, T. J. \& Nieuwdorp, M. Therapeutic potential of fecal microbiota transplantation. Gastroenterology 145, 946-953 (2013).

49. van Nood, E. et al. Duodenal infusion of donor feces for recurrent Clostridium difficile. N. Engl. J. Med. 368, 407-415 (2013).

50. Allen-Vercoe, E. et al. A Canadian Working Group report on fecal microbial therapy: microbial ecosystems therapeutics. Can. J. Gastroenterol. 26, 457-462 (2012).

51. Caporaso, J. G. et al. Moving pictures of the human microbiome. Genome Biol. 12, R50 (2011).

52. US Department of Health \& Human Services. Guidance for industry: enforcement policy regarding investigational new drug requirements for use of fecal microbiota for transplantation to treat Clostridium difficile infection not responsive to standard therapies. US Food and Drug Administration [online], http://www.fda.gov/BiologicsBloodVaccines/ GuidanceComplianceRegulatorylnformation/ Guidances/Vaccines/ucm361379.htm (2013)

53. Petrof, E. O. et al. Stool substitute transplant therapy for the eradication of $C$. difficile infection: 'RePOOPulating the gut'. Microbiome 1, 3 (2013).

54. Petrof, E. O., Claud, E. C., Gloor, G. B. \& Allen-Vercoe, E. Microbial ecosystems therapeutics: a new paradigm in medicine? Benef. Microbes 4, 53-65 (2013). 
55. Schunemann, H., Hill, S., Guyatt, G., Akl, E. A. \& Ahmed, F. The GRADE approach and Bradford §Hill's criteria for causation. J. Epidemiol. Community Health 65, 392-395 (2011).

56. Gordis, L. Epidemiology (W. B. Saunder Company, 2000).

57. European Food Safety Authority. Scientific opinion on the substantiation of health claims related to vitamin $\mathrm{C}$ and reduction of tiredness and fatigue (ID 139, 2622), contribution to normal psychological functions (ID 140), regeneration of the reduced form of vitamin $E$ (ID 202), contribution to normal energy-yielding metabolism (ID 2334, 3196), maintenance of the normal function of the immune system (ID 4321) and protection of DNA, proteins and lipids from oxidative damage (ID 3331) pursuant to Article 13(1) of Regulation (EC) No 1924/2006. EFSA Journal 8, 1815 (2010).

58. Hemila, H. Vitamin $\mathrm{C}$ and the common cold. Br. J. Nutr. 67, 3-16 (1992).

59. Hemila, H. Vitamin $\mathrm{C}$ and common cold incidence: a review of studies with subjects under heavy physical stress. Int. J. Sports Med. 17, 379-383 (1996).

60. Hemila, H. \& Chalker, E. Vitamin C for preventing and treating the common cold. Cochrane Database of Systematic Reviews, Issue 1. Art. No.: CD000980 http:/dx.doi.org/ 10.1002/14651858.CD000980.pub4.

61. Douglas, R. M., Hemila, H., Chalker, E. \& Treacy, B. Vitamin $\mathrm{C}$ for preventing and treating the common cold. Cochrane Database of Systematic Reviews, Issue 3. Art. No.: CD000980 http://dx.doi.org/ 10.1002/14651858.CD000980.pub3.

62. Winzenberg, T. M., Powell, S., Shaw, K. A. \& Jones, G. Vitamin D supplementation for improving bone mineral density in children. Cochrane Database of Systematic Reviews, Issue 10. Art No.: CD006944 http://dx.doi.org/ 10.1002/14651858.CD006944.pub2.

63. Hao, Q., Lu, Z., Dong, B. R., Huang, C. Q. $\& W u, T$. Probiotics for preventing acute upper respiratory tract infections. Cochrane Database of Systematic Reviews, Issue 9. Art. No.: CD006895 http://dx.doi.org/10.1002/ 14651858.CD006895.pub2.

64. Johnston, B. C. et al. Probiotics for the prevention of Clostridium difficile-associated diarrhea: a systematic review and metaanalysis. Ann. Intern. Med. 157, 878-888 (2012).

65. Hempel, S. et al. Probiotics for the prevention and treatment of antibiotic-associated diarrhea: a systematic review and meta-analysis. JAMA 307, 1959-1969 (2012).

66. Hoveyda, N. et al. A systematic review and metaanalysis: probiotics in the treatment of irritable bowel syndrome. BMC Gastroenterol. 9, 15 (2009).

67. Reid, G. Microbiology: Categorize probiotics to speed research. Nature 485, 446 (2012).

68. Sanders, M. E. et al.Probiotics and prebiotics: prospects for public health and nutritional recommendations. Ann. N.Y. Acad. Sci. 1309 19-29 (2014).

69. Hill, C. \& Sanders, M. E. Rethinking "probiotics". Gut Microbes 4, 269-270 (2013).

70. Hoffmann, D. E. et al. Science and regulation. Probiotics: finding the right regulatory balance. Science 342, 314-315 (2013).

71. US Department of Health and Human Services, Food and Drug Administration, Center for Drug Evaluation and Research, Center for Biologics Evaluation and Research \& Center for Food Safety and Applied Nutrition. Guidance for clinical investigators, sponsors, and IRBs. Investigational new drug applications (INDs) - determining whether human research studies can be conducted without an IND. US Food and Drug Administration [online], http://www.fda.gov/downloads/Drugs/ Guidances/UCM229175.pdf (2013).

72. Hungin, A. P. et al. Systematic review: probiotics in the management of lower gastrointestinal symptoms in clinical practice-an evidencebased international guide. Aliment. Pharmacol. Ther. 38, 864-886 (2013).

73. Bertazzoni, E., Donelli, G., Midtvedt, T., Nicoli, J. \& Sanz, Y. Probiotics and clinical effects: is the number what counts? J. Chemother. 25, 193-212 (2013).

74. de Vrese, M. et al. Probiotics-compensation for lactase insufficiency. Am. J. Clin. Nutr. 73, 421S-429S (2001).

75. Guarner, F. et al. Should yoghurt cultures be considered probiotic? Br. J. Nutr. 93, 783-786 (2005).
76. European Food Safety Authority. Introduction of a Qualified Presumption of Safety (QPS) approach for assessment of selected microorganisms referred to EFSA-Opinion of the Scientific Committee http://www.efsa.europa.eu/en/ efsajournal/doc/587.pdf (2007).

77. US Department of Health \& Human Services. Generally Recognized as Safe (GRAS). US Food and Drug Administration [online], http://www.fda.gov/ Food/IngredientsPackagingLabeling/GRAS/ (2013).

78. Higgins, J. \& Green, S. Cochrane Handbook for Systematic Reviews of Interventions (John Wiley \& Sons Ltd, 2008).

79. International Life Sciences Institute-Europe. PASSCLAIM. A European Commission Concerted Action Programme. International Life Sciences Institute [online], http://www.ilsi.org/ Europe/Pages/PASSCLAIM.aspx (2005).

80. GRADE Working Group. Grading the quality of evidence and the strength of recommendations. GRADE [online], http:// www.gradeworkinggroup.org/intro.htm\%20 (2013).

81. van Loveren, H., Sanz, Y. \& Salminen, S. Health claims in Europe: probiotics and prebiotics as case examples. Annu. Rev. Food Sci. Technol. 3 247-261 (2012).

\section{Acknowledgements}

The authors would like to thank members of the ISAPP board of directors who did not directly participate in this consensus panel, but who reviewed, criticized and approved the manuscript: K. Scott, T. R. Klaenhammer, M. Cabana, G. Fahey, E. M. Quigley and J. Ansell.

\section{Author contributions}

All authors made equal contributions to discussion of content and reviewing/editing the manuscript before submission. C.H., F.G., G.R., G.R.G., D.J.M., B.P., L.M. and M.E.S. wrote the article. M.E.S. collated author written contributions into the final text.

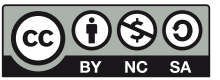

This work is licensed under a Creative Commons AttributionNonCommercial-Sharealike 4.0 Unported License. To view a copy of this license, visit http://creativecommons.org/licenses/by-nc-sa/4.0/. 